\title{
Construction of 3D Shape Models of Femoral Articular Cartilage Using Harmonic Maps
}

\author{
A.D. Brett and C.J. Taylor \\ Division of Imaging Science, Stopford Building, Oxford Road, \\ University of Manchester, Manchester M13 9PT, UK \\ \{a.brett, ctaylor\}@man.ac.uk
}

\begin{abstract}
A previous publication has described a method of pairwise $3 \mathrm{D}$ surface correspondence for the automated generation of landmarks on a set of examples from a class of shape 3. However, that method did not guarantee a diffeomorphic correspondence between examples. This affected the model compactness (the ability of the model to capture shape variation in a small number of parameters) and model specificity (the fact that the model will describe shapes only within the class used for training). In this paper we describe a method of generating the pairwise correspondences using piecewise-linear harmonic maps of the surfaces which is constrained to be diffeomorphic. In particular, we are interested in producing shape models of articular cartilage. In general these models will be close to being planar discs which makes the use of harmonic mapping particularly suitable for our application. An example statistical model built using this new correspondence method is shown for the human femoral articular cartilage; a complex biological shape which demonstrates considerable variation between individuals.
\end{abstract}

\section{Introduction}

The Active Shape Model (ASM) has been shown to provide an accurate and robust segmentation of medical images in 3D [9]. However, construction of these models remains a difficult problem. In $2 \mathrm{D}$, this construction involves the manual identification of a set of $L$ landmarks $\left\{\mathbf{x}_{i} ;(1 \leq i \leq L)\right\}$ for each of $N$ training examples of a class of shapes. A landmark is a point which identifies a salient feature of the shape and which is present on every example of the class. Manual definition of landmarks on 2D shape has proved to be both time-consuming and subjective. The interactive identification of landmarks in 3D images or on pre-segmented surfaces is considerably more difficult and time-consuming than in the $2 \mathrm{D}$ case. In particular, we are interested in producing shape models of articular cartilage, the 3D surface of which has been defined by hand as a set of planar contours. Each contour represents the cartilage outline as it appears in a single $2 \mathrm{D}$ slice of a $3 \mathrm{D}$ sagittal MR image of the knee. The vertices of these sets of contours comprise the pointsets $\left\{D_{i, j} ;(1 \leq i \leq N),\left(1 \leq j \leq n_{D_{i}}\right)\right\}$. The connectivity of each pointset is generated from its contour representation using the algorithm of Geiger [8]. Our motivation for this is to be able to produce corresponded thickness maps 4 of pre-segmented cartilage from 3D MR images. 
A previous publication [3] described a possible solution to the problem of automatic 3D model building. However, the resultant shape model showed folding of the surface due to some groups (triples) of landmarks being re-ordered between training examples. This was as a result of the automated landmarking algorithm not producing a diffeomorphism between the corresponded pairs of shape examples. A diffeomorphism is a mapping which is continuous, one-to-one, onto and differentiable. The effect of this surface folding is on the model compactness (the ability of the model to capture shape variation in a small number of parameters) and model specificity (the fact that the model will describe shapes only within the class used for training). A shape model with a surface which may fold will be both less compact and less specific. Here, we describe a method of generating point correspondences between triangulated surfaces which ensures that the correspondence is diffeomorphic. This method may be incorporated into an algorithm, which is also described here, to produce the corresponded set of examples required to build an ASM.

\section{Related Work}

Kambhamettu and Goldgof [11] and Benayoun et al 1] both propose methods of surface correspondence based on the minimisation of a cost function which involves the difference in the curvature of the surfaces. As pointed out by Tagare [17], curvature is a rigid invariant of shape and its applicability to general nonrigid correspondence is problematic.

Several methods of 3D shape registration have been applied to the problem of building statistical models by producing point correspondences across a training set. Joshi et al [10] deform a template onto hippocampal surface representations using the registration method of Christensen et al [5]. This non-rigid registration uses a course linear elastic matching of volumes followed by refinement by a viscous fluid transform. However, this technique is computationally expensive, requiring a massively parallel computer to solve the partial differential equations of the fluid model. Fleute and Lavalée [7] use an framework of initially matching each training example to a single template, building a mean from these matched examples, and then iteratively matching each example to the current mean and repeating until convergence. Matching is performed using the multi-resolution registration method of Szeliski and Lavalée [16]. This method deforms the volume of space embedding the surface rather than deforming the surface itself. Szekély et al [15] parameterise the surfaces of each of their shape examples using the method of Brechbühler et al [2]. Correspondence may then be established between surfaces but relies upon the choice of a parametric origin on each surface mapping and registration of the coordinate systems of these mappings by the computation of a rotation. 


\section{Harmonic Maps}

A harmonic mapping $h: D \mapsto P$ uniquely maps a (triangulated) topological disk $D$ in $\mathbb{R}^{3}$ to a polygonal region in $\mathbb{R}^{2}$ whilst minimizing metric dispersion. Metric dispersion is a measure of the extent to which small regions in $D$ are stretched in the mapping. In addition, the mapping is independent of the triangulation of $D$, is differentiable on each face of $D$ and is an embedding i.e. it is one-to-one, containing no 'folds' in the planar surface. Because $h: D \mapsto P$ is an embedding, the inverse $h^{-1}$ is a parameterisation of $D$ over $P$. If we imagine that $D$ is constructed from a set of triangular rubber sheets, the harmonic map minimises the total energy $E_{h}$ of this set of rubber sheets.

We construct a piecewise-linear (PL) approximation to a harmonic map using a method described by Eck et al [6]. This method uses an explicit integration of the function $E_{h}$ over each face to reinterpret the problem as the minimisation of the energy of a set of springs, one placed along each edge of $D$. First we form a polygon $P$ by mapping the boundary vertices of $D$ onto a circle in $\mathbb{R}^{2}$. The vertices of $P$ are positioned such that the polygon sides subtend angles proportional to the arc lengths of the boundary segments of $D$. The positions of the rest of the vertices are calculated so that the total energy of the configuration $E_{h}$ is minimised. $E_{h}$ can be interpreted as the energy of a configuration of springs, each spring corresponding to one edge of $D$ :

$$
E_{h}(\mathbf{v})=\frac{1}{2} \sum_{\{i, j\} \in \operatorname{edges}(D)} \kappa_{i, j}\left\|\mathbf{v}_{i}-\mathbf{v}_{j}\right\|^{2}
$$

where $\mathbf{v}_{i}$ is the position of the $i$ th vertex of $D$. The spring constants $\kappa_{i, j}$ are computed as:

$$
\kappa_{i, j}=\frac{\left(l_{i, k_{1}}^{2}+l_{j, k_{1}}^{2}-l_{i, j}^{2}\right)}{A_{i, j, k_{1}}}+\frac{\left(l_{i, k_{2}}^{2}+l_{j, k_{2}}^{2}-l_{i, j}^{2}\right)}{A_{i, j, k_{2}}}
$$

where $l_{i, j}$ is the length of the edge $\{i, j\}$ and $A_{i, j, k}$ is the area of the face $\{i, j, k\}$, both measured in $D$. Each interior edge $\{i, j\}$ is incident to two faces $\left\{i, j, k_{1}\right\}$ and $\left\{i, j, k_{2}\right\}$, the formula for spring constants associated with boundary edges has only one term.

Although $\kappa_{i, j}$ can assume negative values, function (11) is positive definite and its unique minimum can be found by solving the sparse linear least-squares problem $\nabla E_{h}=0$. Following the treatment of Kanai [12, we represent the problem in the form:

$$
E_{h}=\mathbf{V}^{\mathrm{T}} \mathbf{K V}
$$

where $\mathbf{V}=\left(v_{1 x}, v_{1 y}, v_{2 x}, v_{2 y}, \ldots, v_{n x}, v_{n y}\right)$. This is a vector describing all the positions of the vertices in the map. This variable vector can then be separated into two parts, a free part $\mathbf{V}_{\alpha}$ and a fixed part $\mathbf{V}_{\beta}$ representing the boundary vertices. The matrix $\mathbf{K}$ is also divided accordingly:

$$
E_{h}=\left[\begin{array}{ll}
\mathbf{V}_{\alpha}^{\mathrm{T}} & \mathbf{V}_{\beta}^{\mathrm{T}}
\end{array}\right]\left[\begin{array}{ll}
\mathbf{K}_{\alpha \alpha} & \mathbf{K}_{\alpha \beta} \\
\mathbf{K}_{\beta \alpha} & \mathbf{K}_{\beta \beta}
\end{array}\right]\left[\begin{array}{l}
\mathbf{V}_{\alpha} \\
\mathbf{V}_{\beta}
\end{array}\right] .
$$


The minimisation of this energy term has only to be solved over the free parts $\mathbf{V}_{\alpha}$ :

$$
\nabla E_{h}=\frac{\partial E_{h}}{\partial \mathbf{V}_{\alpha}}=\mathbf{K}_{\alpha \alpha} \mathbf{V}_{\alpha}+\mathbf{K}_{\alpha \beta} \mathbf{V}_{\beta}=0 .
$$

Although this is a sparse system, our triangulations and hence $\mathbf{K}$ are not large and we can solve the system using a Cholesky decomposition of the matrix making this a computationally efficient algorithm. Examples of embedded mapping are shown in Figure 1. The mappings of these triangulated meshes took $\sim 2-3$ secs on a $450 \mathrm{MHz}$ P-II.

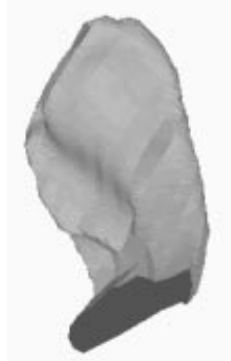

lateral ventricle

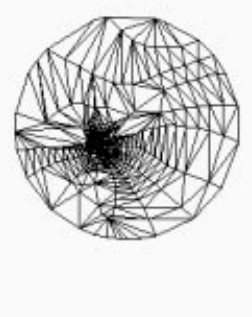

mapping

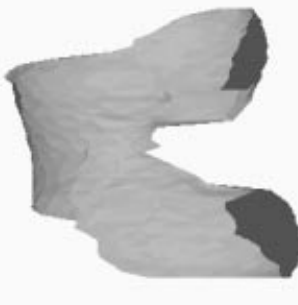

femoral cartilage

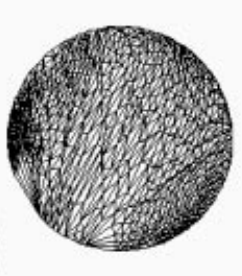

mapping

Fig. 1. A piecewise linear harmonic mapping of a triangulated surface of the frontal part of the left ventricle of the brain and of the upper surface of the femoral articular cartilage. In each case, on the left is a shaded representation of the original dense triangulation with approximately 1000 vertices, on the right is the embedded mapping on the unit circle.

\section{Pairwise Surface Correspondence}

Point correspondences between pairs of surfaces $D_{1}$ and $D_{2}$ may be generated by using their PL-harmonic maps $P_{1}$ and $P_{2}$ in a two-stage algorithm:

1. The two triangulated surfaces $D_{1}$ and $D_{2}$ are registered using a symmetric version of the ICP algorithm which operates on sparse, decimated versions of the surface triangulations. The sets of closest points used for this registration are then used to find the rotation which best registers the PL-harmonic maps $P_{1}$ and $P_{2}$.

2. The positions of the vertices on $P_{1}$ are projected onto $P_{2}$ to give a set of corresponding points on $D_{1}$ and $D_{2}$. Vertex positions on $P_{1}$ are mapped directly to $D_{1}$, the projected vertex positions on $P_{2}$ are mapped onto $D_{2}$ using a barycentric coordinate parameterisation of each triangle.

These two steps will now be described in more detail. 


\subsection{Registration of Maps}

We have used a symmetric version of the Iterative Closest Point (ICP) algorithm [3] to establish registration of the sparse pointset $\left\{D_{1, i}^{\prime \prime}\right\}$ with the dense pointset $\left\{D_{2, i}\right\}$ and of the sparse pointset $\left\{D_{2, i}^{\prime \prime}\right\}$ with the dense pointset $\left\{D_{1, i}\right\}$. The symmetry of this algorithm means that registration is dependant upon matching the salient features (as defined by the decimation algorithm) of both the triangulated surfaces.

Various metrics can be used to define the closest distance between point pairs. We weight the squared distance between points $\left\|X_{i}-Y_{j}\right\|^{2}$ by a factor of $2 /\left(\hat{\mathbf{n}}_{X_{i}}\right.$. $\hat{\mathbf{n}}_{Y_{j}}$ ) where $\hat{\mathbf{n}}_{X_{i}}$ is the unit surface normal on $X$ at point $i$. This encourages the correspondence of points on the surfaces which are topographically equivalent. We label the closest points to $D_{1, i}^{\prime \prime}$ from $D_{2}$ as the pointset $\left\{D_{2, i}^{\prime}\right\}$, and the closest points to $D_{2, i}^{\prime \prime}$ from $D_{1}$ as the pointset $\left\{D_{1, i}^{\prime}\right\}$.

We minimise the registration error such that the pose $Q$ is determined which satisfies:

$$
\begin{array}{r}
E_{D}^{2}=\min _{Q}\left[\frac{1}{n_{D_{1}^{\prime \prime}}} \sum_{i=1}^{n_{D_{1}^{\prime \prime}}}\left\|Q\left(D_{1, i}^{\prime \prime}\right)-Q^{-1}\left(D_{2, b_{i}}^{\prime}\right)\right\|^{2}+\right. \\
\left.\frac{1}{n_{D_{2}^{\prime \prime}}} \sum_{j=1}^{n_{D_{2}^{\prime \prime}}}\left\|Q\left(D_{1, a_{j}}^{\prime}\right)-Q^{-1}\left(D_{2, j}^{\prime \prime}\right)\right\|^{2}\right]
\end{array}
$$

where $Q(\mathbf{p})=s \mathbf{R p}+\mathbf{t}, s$ is a scale factor, $\mathbf{R}$ is a rotation matrix and $\mathbf{t}$ is a translation. The integer values $a_{j}$ index the vertices of $D_{1}^{\prime}$ which are closest to each of the vertices of $D_{2}^{\prime \prime}$ indexed by $j$, and the integer values $b_{i}$ index the vertices of $D_{2}^{\prime}$ which are closest to each of the vertices of $D_{1}^{\prime \prime}$ indexed by $i$. The geometric information of each surface is first normalised such that the centre-ofgravity is at the origin and the mean distance of the points from the origin is 1 . This provides a necessary initial approximation as input to the ICP algorithm which speeds convergence and makes the final solution more robust.

Our sparse triangulation generation algorithm we use makes use of a decimation method described by Schroeder et al [14]. However, we use a different distance metric which we have found to better preserve sharp edges and thin structures. The distance metric, $L$, is computed using Schroeder's distance to mean plane measure as:

$$
L\left(\mathbf{v}_{0}\right)=\left|d\left(\mathbf{v}_{0}\right)-d^{\prime}\left(\mathbf{v}_{0}\right)\right|
$$

where $d\left(\mathbf{v}_{0}\right)$ and $d^{\prime}\left(\mathbf{v}_{0}\right)$ are the signed distances of the vertex $\mathbf{v}_{0}$ to the mean plane of the triangle loop before and after decimation i.e. $d\left(\mathbf{v}_{0}\right)=\hat{\mathbf{u}} \cdot\left(\mathbf{v}_{0}-\overline{\mathbf{x}}\right)$, where $\hat{\mathbf{u}}$ is the unit normal to the mean plane of the triangle loop and $\overline{\mathbf{x}}$ is its centroid. See Figure. 2.

The pointset pairs $\left\{D_{1, i}^{\prime \prime}, D_{2, i}^{\prime}\right\}$ and $\left\{D_{2, i}^{\prime \prime}, D_{1, i}^{\prime}\right\}$ are all positions of vertices on $D_{1}$ and $D_{2}$ and hence can be mapped directly to the vertices of $P_{1}$ and $P_{2}$ as a single pointset pair $\left\{P_{1, i}^{\prime}, P_{2, i}^{\prime}\right\}$ where $P_{1}^{\prime} \subset P_{1}$ and $P_{2}^{\prime} \subset P_{2}$. The maps are 

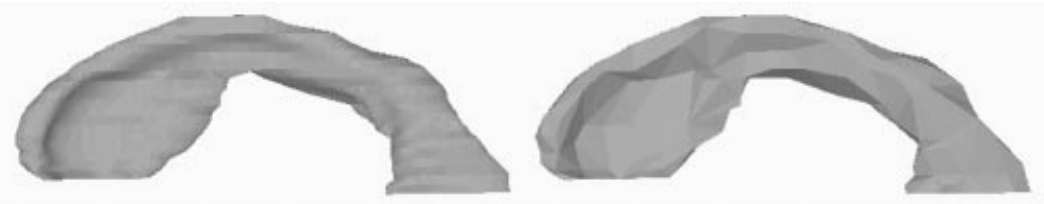

Fig. 2. Result of applying the decimation algorithm to a triangulated surface of the left ventricle of the brain. On the left is a shaded representation of the original dense triangulation with approximately 2000 vertices. On the right the same surface represented by 200 vertices (decimated by $90 \%$ ).

now registered by finding the rotation $\mathbf{R}$ which minimises the term:

$$
E_{P}^{2}=\min _{\mathbf{R}} \sum_{i=1}^{n_{P_{1}^{\prime}}}\left\|P_{1, i}^{\prime}-\mathbf{R}\left(P_{2, i}^{\prime}\right)\right\|^{2}
$$

\subsection{Generating Point Correspondences}

Once the PL-harmonic maps have been registered, we can use them to give corresponding parameterisation of $D_{1}$ and $D_{2}$. For each vertex on $D_{1}$, the mapping of this vertex is found on $P_{1}$ and its position is projected onto the registered version of $P_{2}$. The projection will be in one of three positions with respect to $P_{2}$.

1. Coincident with a vertex position on $P_{2}$. In this case, the mapping to $D_{2}$ is to the vertex position on that surface.

2. Off the edge of the polygon $P_{2}$. The projected point position is simply projected onto the closest boundary edge of $P_{2}$. The mapping onto $D_{2}$ is then made using the positions of the two vertices $\mathbf{v}_{1}$ and $\mathbf{v}_{2}$ which define this closest edge and by the parameterisation of this edge, $s \in[0,1]$, so that the mapped position is: $\mathbf{v}_{m}=\mathbf{v}_{1}+s\left(\mathbf{v}_{2}-\mathbf{v}_{1}\right)$ on $D_{2}$.

3. Within a triangle on $P_{2}$. In this case, the mapping onto $D_{2}$ is made using the positions of the three vertices defining the triangle and three barycentric coordinates which are used to parameterise the triangle so that the point position on $D_{2}$ is given by: $\mathbf{v}_{m}=u \mathbf{v}_{1}+v \mathbf{v}_{2}+w \mathbf{v}_{3}, u+v+w=1$.

This algorithm generates a set of point correspondence pairs between vertex positions of $D_{1}$ and positions on the triangulated surface of $D_{2}$. By matching each example of the training set $\left\{D_{i} ;(2 \leq i \leq N)\right\}$ to $D_{1}$, we can produce a set of corresponding pointsets. We can produce corresponding surfaces by combining the triangulated connectivity of $D_{1}$ with each of these pointsets.

\section{Results}

In order to test the pairwise correspondence algorithm, we have constructed pairs of merged 'mean' shapes, see Figure 3. The algorithms used are computationally 
efficient, the decimation of these $\sim 600$ vertex triangulations to $10 \%$ of their original vertices takes $\sim 5-6$ secs. and the matching phase takes $\sim 20$ sec. on a $450 \mathrm{MHz}$ P-II. A quantitative assessment of the matching and merging of two shape examples is difficult because the 'mean' of two unmatched shape examples is not defined. However, a 'good' merging should preserve the salient features of each example and produce a smooth interpolation of the representation between these. The mean shape can be generated in two ways; by mapping the vertices of $D_{1}$ onto $D_{2}$ or by mapping the vertices of $D_{2}$ onto $D_{1}$. In each case, the final shape is produced by taking the mean of the point positions of the corresponded pair and combing these with the connectivity description (triangulation) of $D_{1}$ or $D_{2}$ respectively. In Figure 3, can we can see that the means generated in these two ways are very similar.

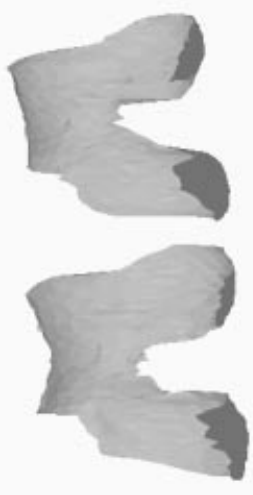

dense surface

upper:

lower:

$$
D_{1}
$$

$D_{2}$
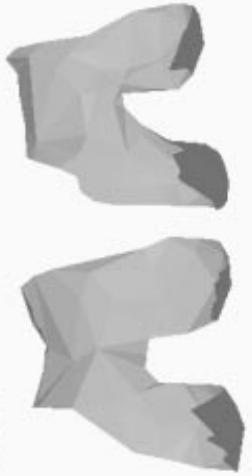

sparse surface

$$
D_{1}^{\prime \prime}
$$

$D_{2}^{\prime \prime}$
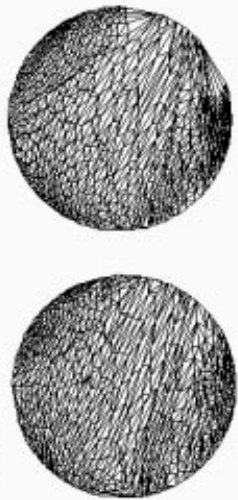

mapping

$P_{1}$

$P_{2}$
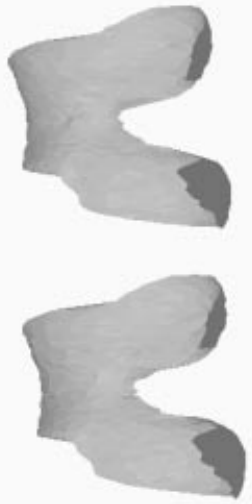

mean

$\left(D_{1}+D_{2}\right) / 2$

$\left(D_{2}+D_{1}\right) / 2$

Fig. 3. Building a 'mean' shape by merging two examples using surface correspondence based on a PL-harmonic mapping. The dense examples are the upper surface of the femoral articular cartilage, defined by a triangulation of $\sim 600$ vertices. The sparse, decimated version are defined by $\sim 60$ vertices. The mean shapes are constructed using mappings of all the vertices of $D_{1}$ on $D_{2}$ (upper) and mappings of all the vertices of $D_{2}$ on $D_{1}$ (lower).

We have also generated a 3D statistical model from six complex biological shapes - the human femoral articular cartilage. These have been defined by hand as contours on a series of 2D slices from 3D Magnetic Resonance images. The example shapes consisted of $\sim 600$ vertices. The first two modes of variation of this

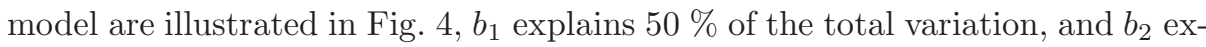
plains $22 \%$. Upon inspection, we can see that the shape instances generated are legal and accurately reflect the shape variation present in the training set of six 
examples - indicating an accurate automated placing of landmark points. There are no tears or folds in the triangulated surfaces of the shape instances which can be caused by crossing or folding of the correspondences between examples.

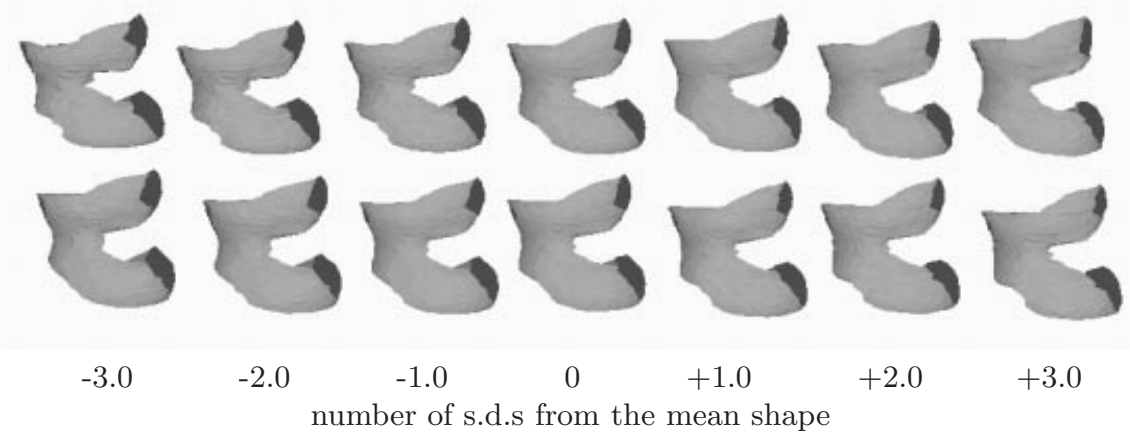

Fig. 4. Shape instances generated using a 3D shape model of six femoral articular cartilage examples. The model consists of $\sim 600$ points. The upper row is illustrates instances of the first mode of shape variation of the model $\left(\mathbf{b}_{1}\right)$, the lower row illustrates instances of the second mode of shape variation of the model $\left(\mathbf{b}_{2}\right)$.

\section{Conclusions}

We have presented a method for the correspondence of two faceted (triangulated) surfaces which are topological discs. The method is based on the parameterisation of the two surfaces by the production of piecewise-linear harmonic maps. We are interested in producing shape models of articular cartilage. In general the examples of this class of shape will be close to being planar discs which have few salient features except at the cartilage edge. This makes the use of harmonic mapping particularly suitable for our application since we assume correspondence between these edges which are the boundaries of our example triangulations.

The harmonic maps are registered by a symmetric form of the ICP algorithm to produce corresponded parameterizations of the pair of surfaces. The use of these maps in the production of corresponding pointset pairs produces a correspondence between the two triangulated surfaces which is diffeomorphic. This pairwise correspondence can be successively applied to a training set to produce a set of landmarked surface examples. We have presented sets of shape instances generated from a 3D shape model built from such a set of landmarks for a set of complex biological shapes. These models have been shown to be compact and to generate legal instances of the set of shapes. We are currently constructing and testing 3D ASMs built using these automatically generated 3D shape models. 
We are aiming to use the harmonic mappings of triangulated surfaces to extend the work on automatic statistical model construction described by Kotcheff and Taylor [13. In that work, a genetic algorithm was used to build 2D statistical models of classes of shape by minimising an objective function based upon the compactness of the resulting model. The optimisation progressed by re-parameterising the boundaries of each of the example shapes to produce a new set of corresponding boundary descriptions at each iteration of the algorithm. Our proposed extension of this work to 3D uses a PL-harmonic mapping of each example as an initial parameterisation of the surface. The model optimisation process is then one of re-parameterising each surface to refine the resulting model.

\section{Acknowledgements}

The authors would like to Dr. Andrew Stoddart for his contribution to the ideas described here. This work was funded by AstraZeneca Pharmaceuticals, Alderley Park, Macclesfield, Cheshire, UK.

\section{References}

[1] A. Benayoun, N. Ayache, and I. Cohen. Adaptive meshes and nonrigid motion computation. In $12^{\text {th }}$ International Conference on Pattern Recognition, pages 730-732, Jerusalem, Israel, 1994.

[2] C. Brechbühler, G. Gerig, and O. Kübler. Parameterisation of closed surfaces for 3-D shape description. Computer Vision, Graphics and Image Processing, 61:154-170, 1995.

[3] A. D. Brett and C. J. Taylor. A method of automated landmark generation for automated 3D PDM construction. Image and Vision Computing, 18(9):739-748, 2000 .

[4] A. D. Brett, J. C. Waterton, S. Solloway, J. E. Foster, M. C. Keen, S. Gandy, B. J. Middleton, R. A. Maciewicz, I. Watt, P. A. Dieppe, and C. J. Taylor. The measurement of focal diurnal variation in the femoral articular cartilage of the knee. In MICCAI'99, pages 328-337, Cambridge, England, Sept. 1999.

[5] G. E. Christensen, S. C. Joshi, and M. Miller. Volumetric transformation of brain anatomy. IEEE Trans. Medical Image, 16:864-877, 1997.

[6] M. Eck, T. DeRose, T. DuChamp, H. Hoppe, M. Lounsberry, and W. Stuetzle. Multiresolution analysis of arbitrary meshes. In Computer Graphics (SIGGRAPH '95 Proceedings), pages 173-182, 1995.

[7] M. Fleute and S. Lavallée. Building a complete surface model from sparse data using statistical shape models: Application to computer assisted knee surgery. In MICCAI, pages 878-887, 1998.

[8] B. Geiger. Three-dimensional modeling of human organs and its application to diagnosis and surgical planning. Technical report no. 2105, Institut National de Recherche en Informatique et Automatique, Dec. 1993.

[9] A. Hill, A. Thornham, and C. J. Taylor. Model-based interpretation of 3D medical images. In J. Illingworth, editor, $4^{\text {th }}$ British Machine Vison Conference, pages 339-348, Guildford, England, Sept. 1993. BMVA Press. 
[10] S. C. Joshi, A. Banerjee, G. E. Christensen, J. G. Csernansky, J. W. Haller, M. I. Miller, and L. Wang. Gaussian random fields on sub-manifolds for characterizing brain surfaces. In J. Duncan and G. Gindi, editors, $15^{\text {th }}$ Conference on Information Processing in Medical Imaging, pages 381-386, Poulteney, VT, 1997. Springer-Verlag.

[11] C. Kambhamettu and D. B. Goldgof. Point correspondence recovery in non-rigid motion. In IEEE Conference on Computer Vision and Pattern Recognition, pages 222-227, 1992.

[12] T. Kanai, H. Suzuki, and F. Kimura. Three-dimensional geometric metamorphosis based on harmonic maps. The Visual Computer, 14:166-176, 1998.

[13] A. C. W. Kotcheff and C. J. Taylor. Automatic construction of eigenshape models by direct optimization. Medical Image Analysis, 2(4):303-314, 1998.

[14] W. J. Schroeder, J. A. Zarge, and W. E. Lorensen. Decimation of triangle meshes. Computer Graphics, 26(2):65-70, 1992.

[15] G. Székely, A. Kelemen, C. Brechbühler, and G. Gerig. Segmentation of 2-d and 3 -d objects from mri volume data using constrained elastic deformations of flexible fourier contour and surface models. Medical Image Analysis, 1:19-34, 1996.

[16] G. Szeliski and S. Lavalée. Matching 3-D anatomical surface with non-rigid deformations using octree-splines. International Journal of Computer Vision, 18(2):171-186, 1996.

[17] H. D. Tagare. Shape-based nonrigid correspondence with application to heart motion analysis. IEEE Transactions on Medical Imaging, 18(7):434-439, 1999. 